\title{
Bi-stable RF-MEMS Switched Capacitor Based on Metal-to-Metal Stiction
}

\author{
Achref Yahiaoui, Arnaud Pothier, Pierre Blondy \\ XLIM UMR 6172 - Université de Limoges/CNRS, 123 Avenue Albert Thomas, 87060 Limoges France
}

\begin{abstract}
This paper presents a new concept for the realization of a bi-stable RF-MEMS switched capacitor using a resistive contact. The main idea is to maintain the device in a given position using metal-to-metal stiction. The metal-to-metal contact is used only for mechanical purposes and has no electrical function. 20 Volts, $10 \mu$ sec pulses are used to switch the device from one stable position to the other. After disconnection, the device maintains its position with extremely little change over long periods. $0.06 \%$ on-state capacitance relative shift has been measured over 4 days with daily control, and lab environment closet storage. 5 minutes periodic cycling shows very little drift in both states of the RF-MEMS capacitor. Moreover, the device is fabricated using MEMS conventional processing steps permits to obtain capacitive contrast of 3 .
\end{abstract}

Index Terms - RF-MEMS, variable capacitors, metal contact, switched capacitors.

\section{INTRODUCTION}

Improvement of the RF-MEMS industrial maturity makes this technology more and more attractive for reconfigurable systems, such as switching networks, portable wireless systems, phased arrays, low-noise oscillators and amplifiers [1]. This is because of the very low loss and the outstanding linearity of RF-MEMS capacitors arrays. In general, RFMEMS switched components are using electrostatic actuation to deflect metallic suspended beams. Such systems are designed so that the beam returns to its initial position once the electrostatic bias is removed. The operation is similar to switched capacitor based on semi-conductor technology, and requires permanent bias.

At microwave frequencies, bi-stable components, like floating-gate devices have very low $\mathrm{Q}$, and mechanical devices, such as relays, or stepped motors are generally slow and bulky. A chip-sized, high Q bi-stable device would find numerous applications at microwave frequencies, since it would permit the simplification of complex control systems, and simplify applications like electronically steerable of antenna arrays.

For instance, a bi-stable RF-MEMS capacitor would permit $\mathrm{X}-\mathrm{Y}$ successive addressing of tunable element in reflect array antennas, or ground tuning of tunable filters for space applications, exactly like with stepped motors but at a small fraction of size and weight.

This paper introduces a novel switched capacitor that uses metal-to-metal stiction for mechanical purpose only, in a similar fashion as the concept presented in [1], with a specific design for RF. In the mentioned reference, metal-to-metal stiction has permitted to reach more than ten billion of cycles, using a very clean packaging, for static memory applications.

This paper will first present the general properties of metal contacts, and then a bi-stable mechanical structure based on this effect. RF performances will be presented and first stability testing for several days.

\section{Properties of Metal to Metal Contact}

Fig. 1 shows typical force-contact resistance diagram of a metal-to-metal contact [2]. When two metals are brought into contact, as the applied force increases, the contact resistance decreases due to metal inter-penetration (called the contact interference). To open the contact, the metals have to be pulled apart, and metal contact diagrams present hysteresis. It means that two metals brought in contact will remain in contact if no separation force is applied. Material properties have large influence on the force/resistance ratio and hysteresis. Soft metals like gold result in large contact area, and large hysteresis. Harder metals like chromium or even Ruthenium, result in a smaller contact area, and little or no hysteresis.

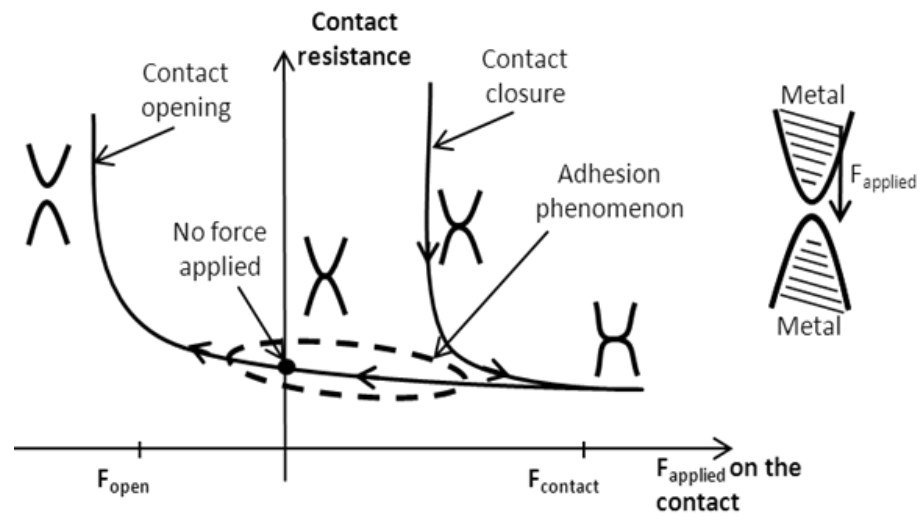

Fig. 1. Typical force to contact resistance diagram of a metal contact. The diagram clearly shows the presence of hysteresis allowing for mechanical bi-stability.

\section{OPERATION PRINCIPLE}

A cross section of the proposed component is shown in Fig. 2 outlines the operation of the device. The RF-MEMS device is made with a suspended tilting plate, that includes a center pivot dimple, a metal stiction dimple, and a capacitance area. The metal plate is initially suspended with its center pivot dimple above 2 electrodes (Fig. 2 position (a)). By applying a 
voltage in one of two electrodes, the beam is attracted by one of its two sides and ends into one of the position (c) or (e). Because of metal stiction in the contact zone, the device remains in this position (position (d) or (f)) without any bias applied. Passing from one position to the other can be done by applying a voltage pulse on the opposite side of the contact zone.

(a)

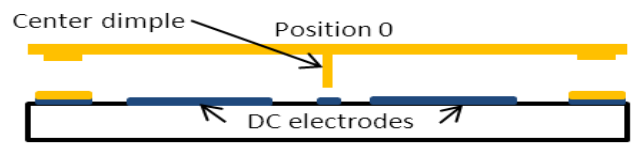

(b)

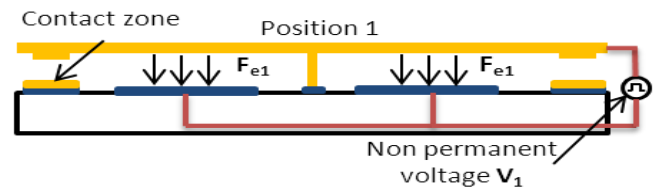

(c)
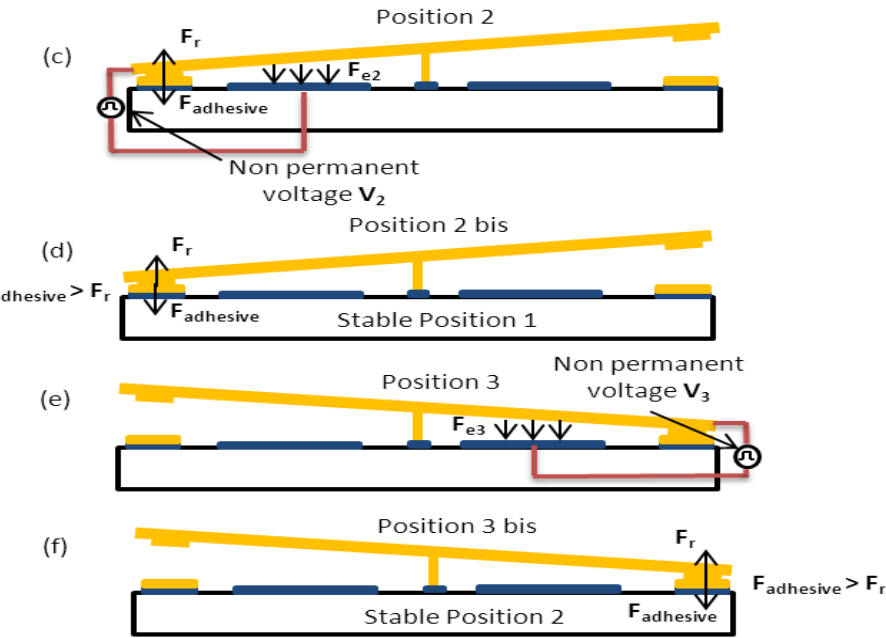

Fig. 2. Cross section view of the four operations steps of the proposed device. (a) is the initial state, (b) is the intermediate stable state, (d) and (f) are the two possible stable states of the device.

\section{DESIGN RF-MEMS AND MECHANICAL ANALYSIS}

The bi-stable switched capacitor is based on a $150 \times 160 \mu \mathrm{m}^{2}$ suspended plate separated from two $35 \times 160 \mu \mathrm{m}^{2}$ actuation electrodes by a $1.4 \mu \mathrm{m}$ gap (see Fig. 3). The $1.4 \mu \mathrm{m}$ gap is made with a two steps sacrificial layer, allowing $0.3 \mu \mathrm{m}$ separation in the pivot dimple area and $1.1 \mu \mathrm{m}$ in the contact area. Long and meandered suspension arms reduce the sensitivity to axial stress and permit rotation movement around the center pivot dimple. The rotation stiffness is extremely low, and electrostatic forces dominate movement. The center pivot dimple contact is made with $\mathrm{Au}$ to $\mathrm{Cr}$ contact, that has very low adhesion forces.

After release, the structure presents a vertical stiffness 20 $\mathrm{N} / \mathrm{m}$ enough to keep it suspended. Then, when a 5 Volts nonpermanent DC actuation is applied between the beam and the two electrodes, the plate is comes in contact with the substrate. To pivot the beam from this position to one of two sides, a 3 Volts pulse (10 $\mu \mathrm{s}$ width) is applied on one of two electrodes (Fig. 2 position (c) or (e)). This result in $\mathrm{Au}-\mathrm{Au}$ contact between the $0.3 \mu \mathrm{m}$ dimple located at the contact zone. In this configuration, the simulated restoring force $F_{r 2}$ is in the order of $3.6 \mu \mathrm{N}$ just enough to stick the beam in this position. The measured voltage able to break the metal contact is 4 Volts. Thus, based on a moment calculation, the deduced minimum adhesive force $\mathrm{F}_{\text {adhesive }}$ is $3.7 \mu \mathrm{N}$.

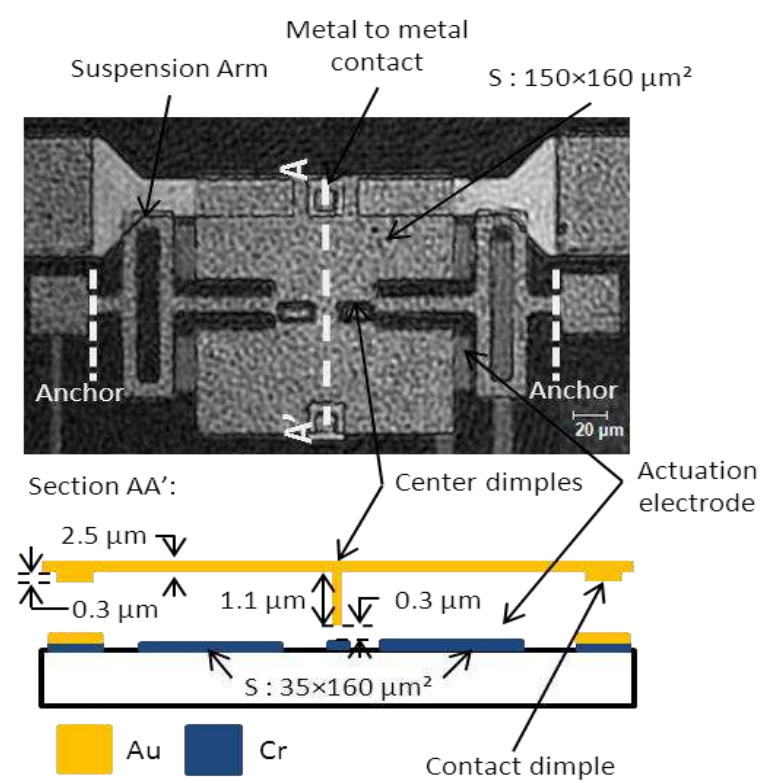

Fig. 3. Microphotograph and cross section view of the bi-stable RFMEMS switched capacitor.

For proper operation, the plate is actuated with DC pulses (Magnitude: 20 Volts, and width: $10 \mu \mathrm{s}$ ) to toggle the plate from position 2 (Fig. 2. d) to position 3 (Fig. 2. f) and vice versa.

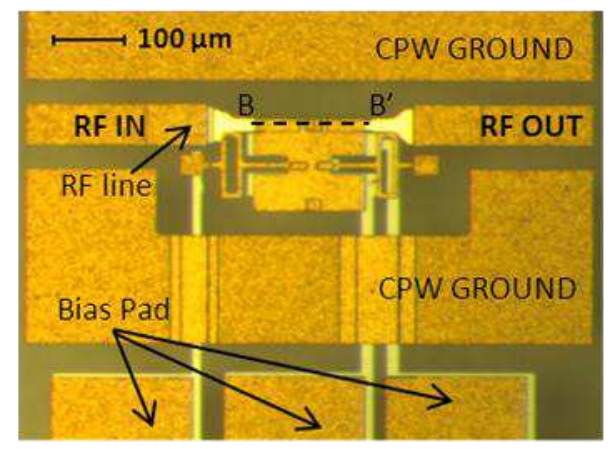

Section $\mathrm{BB}^{\prime}$ :

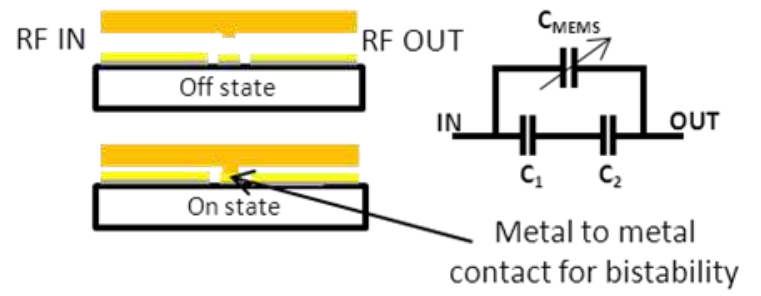

Fig. 4. Microphotograph, section view and electrical equivalent scheme of the fabricated device. 


\section{RF PERFORMANCE AND RELIABILITY TEST}

\section{A. RF performance}

The device was measured on a cascade microwave probe station in laboratory environment. The fitted $\mathrm{C}_{\text {on }}$ capacitance and $\mathrm{C}_{\text {off }}$ capacitance are $15 \mathrm{fF}$ and $4.8 \mathrm{fF}$ respectively, giving a capacitive ratio of 3 . As shown in Fig. 5, the capacitance values are very small, and the measured reflection coefficient is very high, indicating that the devices have extremely high Q.

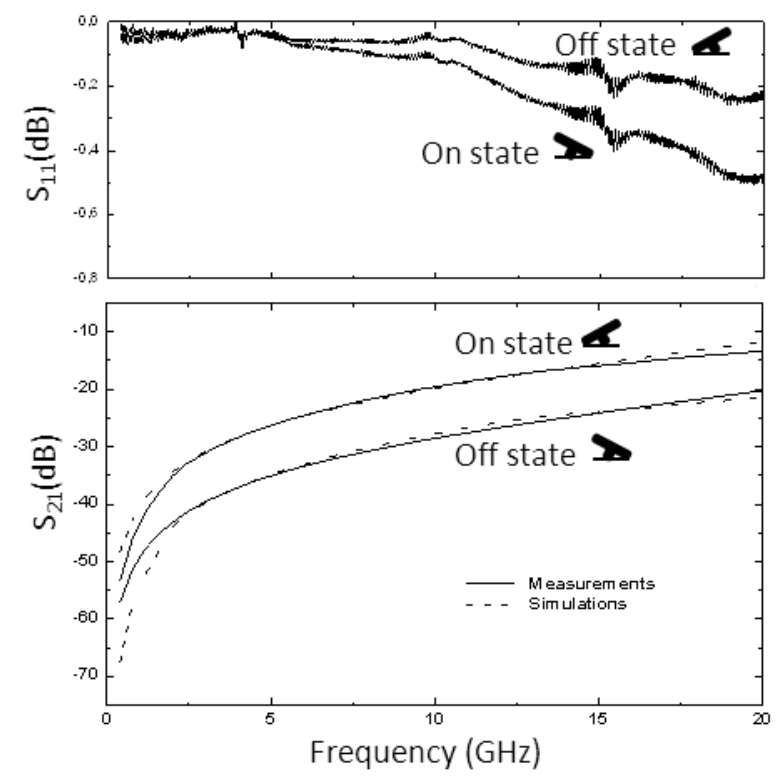

Fig. 5. Measured $\mathrm{S}$ parameters of the device in its $\mathrm{ON}$ and $\mathrm{OFF}$ states, corresponding to Fig. 1(d) and (f).

\section{B. Stability testings}

To validate this concept, a device was switched from ON to OFF position evry 5 minutes (Fig. 6. a) in open laboratory environment. The device is measured in a given state, then toggled to the other state, and measured after bias has been removed. It can be seen that the ON to OFF state capacitance remains the same, indicating the bi-stabilty of the device.

At the end of this periodic cycling test, the device has been left in ON position for 4 days in non vaccuum condition (Fig. 6. b). The shifting of $\mathrm{C}_{\text {on }}$ capacitance os in the order of $0.06 \%$. However the DC voltage required to break the metal contact passed from 4 Volts to 7 Volts. A non-permanent DC voltage was applied to toggle to the off side in order to have a good adhesive force and the device was left in this Off position in non vacum condition for 24 hours, $6 \%$ of shifting was noted. That can be explained by the fact that the tip not on contact is more sensitive tout a gradient of stress.



(a)

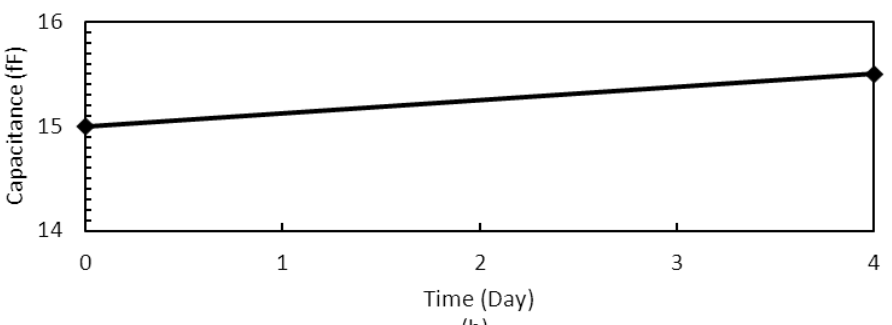

(b)

Fig. 6. Stability testing, (a) periodic cycling, (b) $\mathrm{C}_{\text {on }}$ evolution over 4 days.

\section{CONCLUSION}

This paper presented a new concept of bistable RF-MEMS switched capacitor. The big interest such system is to keep the device on a particular position without once the bias is removed, only by basing on adhesive forces. The device was designed to present very low restoring forces in each position. The capacitance contrast of 3 is obtained only by the gap ration and no dielectric layer was used.

The concept demonstrated a good stability of $\mathrm{C}_{\text {on }}$ and $\mathrm{C}_{\text {off }}$ capacitance for periodic cycling. This stability was approved further by measuring the shifting of $\mathrm{C}_{\text {on }}$ capacitance after leaving the device on its $\mathrm{ON}$ state for 4 days in non-vacuum condition. That revealed a shifting of $0.06 \%$.

Work is on-going in our laboratory for more reliability testing and switching time measurement. In a long term view, enhance capacitance contrast by using dielectric layer.

\section{REFERENCES}

[1] V. Joshi , C. Smith, R. Gaddi , D. Lacey , T. Nagata , M. Renault, A. Unamuno, R. V. Kampen, R. Knipe and D. Yost "A non volatile MEMS switch for harsh environment memory applications", Proc. 10th Annu. Non-Volatile Memory Technol. Symp., pp.6-2 2009.

[2] J.B. Pethica and D. Tabor « Contact of Characterised Metal Surfaces at very Low Loads: Deformation and Adhesion », Surface Science, 89 (1979), pp 182-190. 